\title{
Size mediated control of the optical and magneto-optical properties of Co nanoparticles in $\mathrm{ZrO}_{2}$
}

\author{
C. Clavero, ${ }^{\text {a) }}$ B. Sepúlveda, and G. Armelles \\ Instituto de Microelectrónica de Madrid (IMM) (CNM-CSIC), Isaac Newton 8-PTM, \\ E-28760 Tres Cantos, Madrid, Spain
}

Z. Konstantinović, M. García del Muro, A. Labarta, and X. Batlle

Departament de Física Fonamental, Universitat de Barcelona, Marti i Franquès 1, 08028 Barcelona, Spain and Institut de Nanociència i Nanotecnologia, Universitat de Barcelona, Martí i Franquès 1, 08028 Barcelona, Spain

(Received 30 May 2006; accepted 12 July 2006; published online 12 October 2006)

\begin{abstract}
We present a study of the optical and magneto-optical (MO) properties of Co nanoparticles embedded in $\mathrm{ZrO}_{2}$ in the spectral range from 1.4 to $4.3 \mathrm{eV}$. The nanostructured films were prepared by pulsed laser deposition in a wide range of Co nanoparticle concentrations varying from $20 \%$ to $80 \%$. For Co concentration lower than $x \sim 0.45$ the size of the nanoparticles was found to remain almost constant $(D \sim 2.5 \mathrm{~nm})$, whereas it increases above it. Differences are found between the optical and MO constant of the Co nanoparticles and those of continuous Co films. Those differences are associated with size effects of the intraband contribution inside the nanoparticles. (C) 2006 American Institute of Physics. [DOI: 10.1063/1.2356799]
\end{abstract}

\section{INTRODUCTION}

Granular films consisting of Co nanoparticles embedded in a dielectric matrix are attracting a great scientific and technological interest due to their physical properties that differ considerably from the bulk properties of the constituent materials and that can be used in a large number of applications. These differences are due to the fact that in the nanoparticles a relatively large fraction of the atoms are placed in the surface, and also from their finite size that may produce quantum size effects. ${ }^{1-4}$ Some of the interesting properties and applications of these granular materials are the enhanced Kerr rotation, ${ }^{5}$ useful in magneto-optic (MO) recording media $^{6}$ and sensors, or the giant magnetoresistance due to spin-dependent tunneling, ${ }^{7,8}$ applicable for sensors and read heads. In addition, their high coercivity, ${ }^{9,10}$ permeability, and electrical resistivity are valuable for shielding and bit writing at high frequencies in magnetic recording media. ${ }^{11}$

In this paper we present a study of the optical and MO response of Co nanoparticles embedded in $\mathrm{ZrO}_{2}$. Using an effective medium approximation, we obtain the optical and MO properties of the Co nanoparticles, which differ from those of Co bulk material and depend on the size of the nanoparticles. After a brief description of the fabrication and experimental characterization techniques in Sec. II, we describe in Sec. III the morphological and structural evolution of the Co nanoparticles as a function of the Co concentration. In Sec. IV the magnetic response of the samples is presented and in Sec. V the spectral response of the samples is calculated and described. Finally in Sec. VI we discuss how the optical and MO response of the system are influenced by the size of the nanoparticles.

\footnotetext{
a) Author to whom correspondence should be addressed; electronic mail: cesarc1@imm.cnm.csic.es
}

\section{EXPERIMENT}

$\mathrm{Co}-\mathrm{ZrO}_{2}$ granular films were grown in a high vacuum chamber equipped with a pulsed laser deposition (PLD) system. The $200 \mathrm{~nm}$ thick films were deposited on Si substrates by $\mathrm{KrF}$ PLD (wavelength of $248 \mathrm{~nm}$, pulse duration $\tau$ $=34 \mathrm{~ns})$ at room temperature (RT) and with Co volume concentrations ranging from $20 \%$ to $80 \%$. Rotating composite targets made of sectors of $\mathrm{ZrO}_{2}$ and pure Co were used. Different surface ratios of target components led to different volume fractions of Co. The target-substrate distance was fixed at $30 \mathrm{~mm}$ and the laser fluency was typically $3 \mathrm{~J} / \mathrm{cm}^{2}$. Three different granular samples were prepared with Co concentrations of $20 \%, 45 \%$, and $80 \%$, respectively. The average composition and thickness were determined by microprobe analyses. The structure of the samples was inspected by $\mathrm{x}$-ray diffraction (XRD) and high-resolution transmission electron microscopy (HRTEM). Particle size distributions were obtained from TEM. This parameter was also calculated by fitting the low-field magnetic susceptibility in zerofield cooling (ZFC) and the high temperature isothermal magnetization curves, $M(H)$, to a distribution of Langevin functions which model the superparamagnetic behavior of the particle size distribution. In ZFC experiments the sample was cooled down to the lowest measuring temperature ( $2 \mathrm{~K}$ ) and then a 50 Oe field was applied, being the magnetization measured as temperature is increased. The magnetization curves $M(H)$ were measured up to $50 \mathrm{kOe}$.

The optical characterization was carried out by measuring the ellipsometric parameters $\tan \Psi$ and $\cos \delta$ with a spectroscopic rotating polarized ellipsometer in the $1.4-4.2 \mathrm{eV}$ spectral range, at both $50^{\circ}$ and $70^{\circ}$ incidence angles. The magnetic and $\mathrm{MO}$ properties were studied using a polar Kerr MO spectrometer described elsewhere ${ }^{12}$ in the spectral range from 1.4 to $4.3 \mathrm{eV}$. In the polar configuration the magnetic 


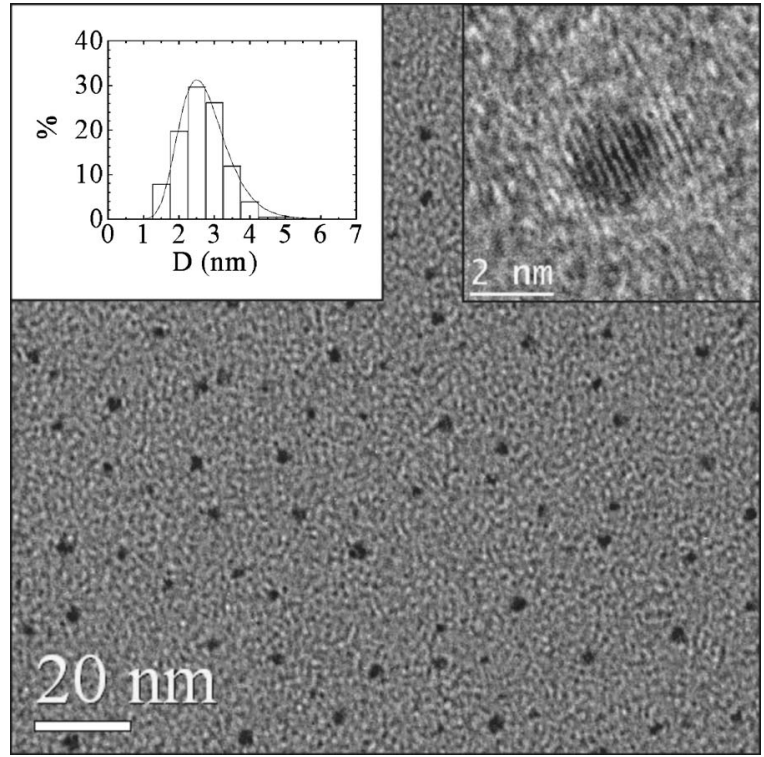

FIG. 1. TEM images of a $\mathrm{Co}-\mathrm{ZrO}_{2}$ granular film with $20 \%$ Co. Right-handside inset shows the magnification of one nanoparticle. Left-hand-side inset shows the histogram of the size distribution obtained from the TEM micrograph.

field is applied perpendicular to the plane of the sample, the angle of incidence is $5^{\circ}$, and the applied magnetic field is $1.6 \mathrm{~T}$.

\section{MORPHOLOGY AND STRUCTURE}

The structure of the samples was investigated by means of HRTEM, XRD, and microprobe. Figure 1 shows a bright field TEM micrograph corresponding to the $\mathrm{Co}-\mathrm{ZrO}_{2}$ thin film with $20 \%$ Co concentration. The dark regions indicate the Co nanoparticles whereas the light regions correspond to amorphous $\mathrm{ZrO}_{2}$. It can be clearly observed how Co is forming a regular distribution of isolated nanoparticles embedded in the $\mathrm{ZrO}_{2}$ matrix. The right-hand-side inset to Fig. 1 shows the magnification of a nanoparticle, where the lattice fringes corresponding to Co atomic planes can be clearly seen. The nanoparticles exhibit very well defined interfaces with the matrix, and their size distribution can be fitted to a linearlogarithmic function with a mean particle size of $D$ $=2.5 \mathrm{~nm}$ (see left-hand-side inset to Fig. 1). The size of the nanoparticles was found to remain almost constant below the percolation limit. ${ }^{8}$ In the case of the $45 \%$ sample TEM micrographs show that the neighboring nanoparticles start to touch each other, giving rise to randomly oriented chains, which manifests the proximity of the percolation threshold.
Finally, in the sample with the highest Co concentration (80\%), Co aggregates form a continuum medium containing a dispersion of amorphous $\mathrm{ZrO}_{2}$ clusters. The observed mean sizes and their growth with increasing Co concentration are less pronounced as compared with $\mathrm{Co}-\mathrm{MgO},{ }^{13} \mathrm{Co}-\mathrm{SiO}_{2},{ }^{14}$ or $\mathrm{Co}-\mathrm{Al}_{2} \mathrm{O}_{3}$ (Ref. 15) granular systems prepared by sputtering. This behavior may be related to the choice of the matrix oxide. The $\mathrm{ZrO}_{2}$ seems to coat the Co nanoparticles confining them in small globules, thus avoiding the growth of large clusters.

Diffraction pattern from TEM suggests that for low Co content, the structure of the nanoparticles is face-centeredcubic (fcc), as observed in the literature. ${ }^{16,17}$ For concentrations above 0.40 , the majority of the crystalline structure is still in fcc, but the XRD spectra indicate also the existence of the hexagonal-close-packed (hcp) phase.

\section{MAGNETIC RESPONSE}

The magnetic behavior of the samples was inspected by measuring polar Kerr loops and ZFC and magnetization curves. In the case of polar Kerr loops a magnetic field was applied perpendicular to the sample and the Kerr rotation angle of the light (proportional to the magnetization) was registered. The loops corresponding to the $20 \%, 45 \%$, and $80 \%$ samples are shown in Fig. 2. Closed loops are found, corresponding to a system with in plane magnetization in all the samples. The evolution of the loops and the gradual increase of the saturation field reveal the transition from a system with small magnetically isolated nanoparticles to a percolated system with large aggregates (as observed by TEM). ${ }^{18}$

More information about the magnetic response of the system related to the morphology can be obtained by measuring ZFC susceptibility and isothermal magnetization curves below the percolation (20\% sample). A narrow particle size distribution can be determined by fitting the ZFC susceptibility and $M(H)$ curves in the superparamagnetic region to a distribution of Langevin functions. ${ }^{8}$ In these calculations the particles are considered to have a linearlogarithmic size distribution. The obtained parameters of the particle distribution are a mean particle size $D=2.5 \mathrm{~nm}$ and a width of $\sigma=0.12$ for $x=0.20$, which directly matched TEM observations.

\section{SPECTRAL RESPONSE}

The optical constants corresponding to the three different samples are depicted in Fig. 3. These results are compared to

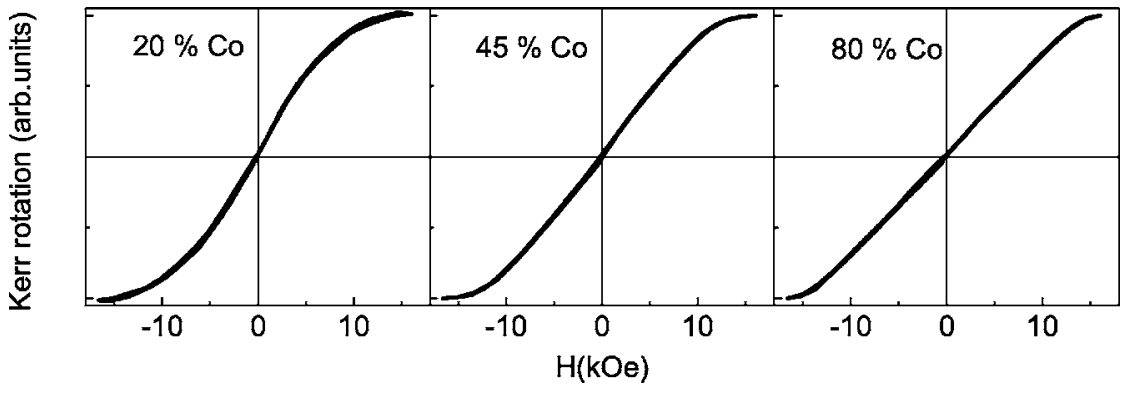

FIG. 2. Polar Kerr loops corresponding to the $20 \%, 45 \%$, and $80 \%$ Co samples. 


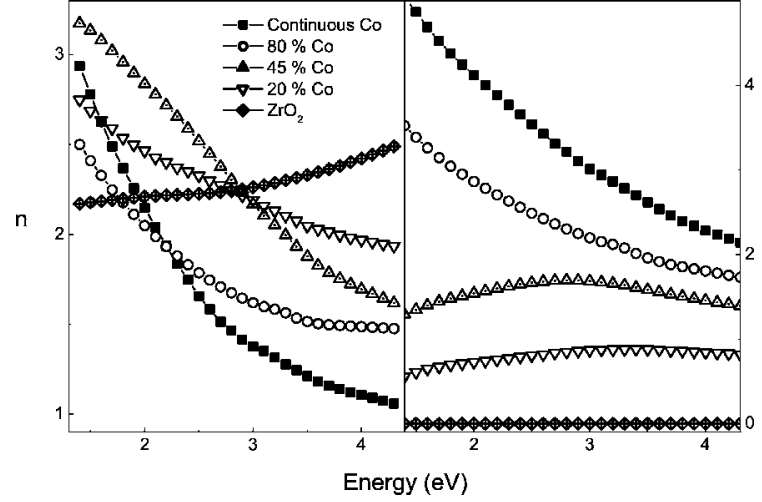

FIG. 3. Measured real $n$ and imaginary $k$ parts of the refractive indices corresponding to the films containing $20 \%, 45 \%$, and $80 \%$ of Co nanoparticles. The indices of a polycrystalline Co film and of $\mathrm{ZrO}_{2}$ are depicted for comparison.

the optical constants of $\mathrm{ZrO}_{2}$ and of a polycrystalline thick continuous Co film grown in the same conditions. As can be observed, the $80 \%$ sample exhibits a real part of the refractive index $(n)$ curve similar to bulk Co, with a slight evolution towards the $\mathrm{ZrO}_{2}$ value, and a reduction of the imaginary part of the refractive index $(k)$ due to the presence of transparent $\mathrm{ZrO}_{2}$. In the case of the $45 \%$ and $20 \%$ samples the evolution of $n$ towards the $\mathrm{ZrO}_{2}$ value can be noted as an increase of the intensity and a change in the slope of the curves, accompanied with a progressive reduction of $k$ as the Co concentration decreases.

To obtain the MO constants of the samples we have measured the Kerr rotation and ellipticity spectra of the samples (Fig. 4). As can be observed, the rotation increases as the Co concentration rises, whereas a less gradual evolution is found in the ellipticity spectra. In Fig. 5 we present the MO constant of the samples. These values were obtained from the Kerr rotation spectra (Fig. 4) and optical constant of the layers (Fig. 3) using a transfer matrix formalism. ${ }^{19,20}$ The MO constants of a thick polycrystalline Co film are also shown in Fig. 5. As can be observed, as Co content decreases, there is a strong diminution of the value of the real part of the MO constant, especially in the visible and the near-infrared spectral region, with a change of the sign for the $45 \%$ and $20 \%$ samples. On the contrary, the decrease of

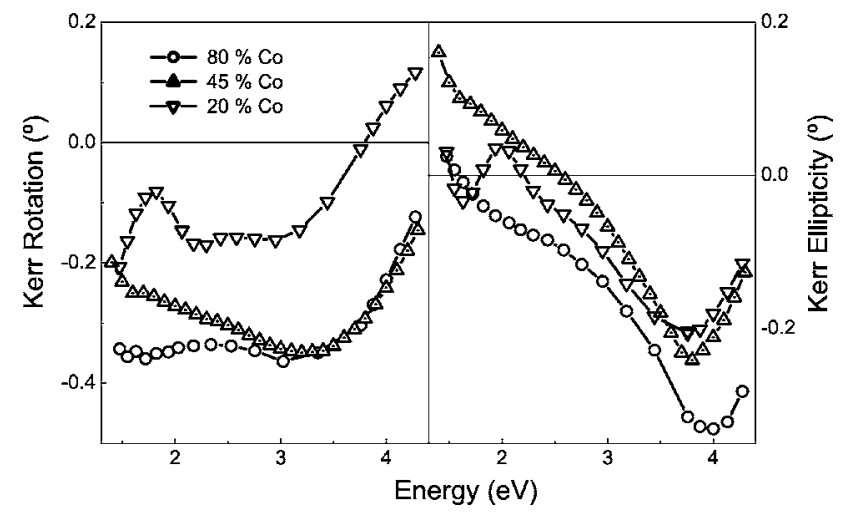

FIG. 4. Polar Kerr rotation and ellipticity for the $20 \%$, $45 \%$, and $80 \%$ Co samples.

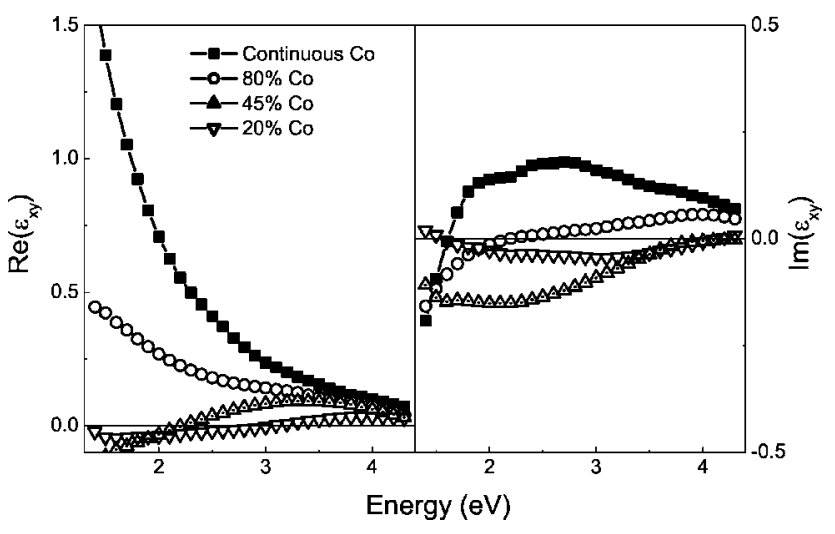

FIG. 5. Real and imaginary parts of the off-diagonal components of the dielectric tensor for the $20 \%, 45 \%$, and $80 \%$ granular films accompanied with those extracted from a polycrystalline Co film.

the intensity of the imaginary part is less pronounced, with also a change of the sign for the $45 \%$ and $20 \%$ samples.

The variation of the optical properties of granular media may be due to the change of the optical and MO constants of the Co particles located inside the layers with respect to the Co polycrystalline film. Since the sizes of the Co nanoparticles inside the layers are much smaller than the wavelength of the incident light we can use an effective medium approximation $^{21}$ that takes into account the Co concentration dependence to obtain the optical and MO properties of the Co located inside the layers. In the Maxwell-Garnet approximation the effective dielectric tensor $\varepsilon_{e}$ of a composite material consisting of particles randomly distributed in a matrix depends on the dielectric tensors of the particles and matrix, and concentration and shape of the particles ${ }^{21-23}$ as follows:

$$
\begin{aligned}
& \varepsilon_{x x}^{\text {eff }}=\varepsilon_{x x}^{m}+\frac{f_{p}\left(\varepsilon_{x x}^{p}-\varepsilon_{x x}^{m}\right)}{a}, \\
& \varepsilon_{x y}^{\text {eff }}=\frac{f_{p} \varepsilon_{x y}^{p}}{a^{2}}, \\
& a=\left[1+(1-f) \frac{\left(\varepsilon_{x x}^{p}-\varepsilon_{x x}^{m}\right)}{\varepsilon_{x x}^{m}} L_{x}\right],
\end{aligned}
$$

where $\varepsilon_{x x}^{p}, \varepsilon_{x y}^{p}, \varepsilon_{x x}^{m}, \varepsilon_{x x}^{\text {eff }}$, and $\varepsilon_{x y}^{\text {eff }}$ are the optical and MO constants of the nanoparticles, matrix, and effective medium, respectively, $f$ is the nanoparticle concentration, and $L_{x}$ is the component of the depolarization tensor, which depends on the shape of the nanoparticle. ${ }^{22}$ Note that the concentration of nanoparticles must be lower than 50\%; if it is higher it must be considered as the matrix element. Therefore, from the measured optical and MO constant of the layers (Figs. 3 and 5) and assuming that the nanoparticles have spherical shape (as observed by TEM), we can obtain the diagonal [Fig. 6(a)] and off-diagonal [Fig. 6(b)] components of the dielectric tensor of the Co inside the three different layers. As can be observed the diagonal components of the dielectric tensor of Co corresponding to the different samples depend on the Co concentration. In particular, we observe a decrease of the intensity of the real part as we decrease the Co concentration whereas smaller changes are observed in the 
imaginary part. On the other hand, the off-diagonal components of Co exhibits a different behavior: the $80 \%$ sample's Co has values similar to those of polycrystalline bulk Co, whereas the values of Co in the $45 \%$ and $20 \%$ samples are very similar between them and different from those of the $80 \%$ sample. In fact, in the $20 \%$ and $45 \%$ samples the Co nanoparticles have very similar sizes, whereas in the $80 \%$ sample the nanoparticles percolate. These results suggest a different size dependence for the diagonal and off-diagonal components of the Co dielectric tensor.

\section{DISCUSSION}

The dielectric constant of metallic systems has two predominant contributions: intraband (owing to conduction electrons in the material) and interband contributions (due to interband transitions):

$$
\varepsilon(\omega)=\varepsilon^{\text {interband }}(\omega)+\varepsilon^{\text {intraband }}(\omega) .
$$

The intraband contribution can be described using the Drude model $^{24}$ as follows: ${ }^{25}$

$$
\begin{aligned}
& \varepsilon_{x x}^{\text {intraband }}(\omega)=1+i \frac{(1-i \omega \tau) \omega_{p}^{2} \tau}{\omega\left[(1-i \omega \tau)^{2}+\left(\omega_{c} \tau\right)^{2}\right]}, \\
& \varepsilon_{z z}^{\text {intraband }}(\omega)=1+i \frac{\omega_{p}^{2} \tau}{\omega(1-i \omega \tau)}, \\
& \varepsilon_{x y}^{\text {intraband }}(\omega)=\frac{\omega_{c}\left(\omega_{p} \tau\right)^{2}}{\omega\left[(1-i \omega \tau)^{2}+\left(\omega_{c} \tau\right)^{2}\right]},
\end{aligned}
$$

where $\omega_{p}=\sqrt{4 \pi n e^{2} / m}$ is the plasma frequency, $\omega_{c}=e B / m c$ is the cyclotron frequency, and $\tau$ is the relaxation time of the electrons, which depends on the electron-electron, electronphonon, and electron-defect scattering contributions. ${ }^{2}$

The wavelength dependence of diagonal components of Co cannot be described taking into account only the expression given in Eq. (3) due to intraband contributions. On the other hand, we can use those expressions to represent the wavelength dependence of the nondiagonal components of Co, as is shown in Fig. 6. The thick continuous lines in Figs. 6(a) and 6(b) represent the calculated MO constants given by Eq. (3) with the parameters obtained for polycrystalline Co by Krinchik $^{26,27} \quad\left(\omega_{p}=9.74 \mathrm{eV}, \quad \omega_{c}=0.089 \mathrm{eV}, \quad\right.$ and $\tau$ $=0.632 \mathrm{eV}^{-1}$ ). As can be observed, with these parameters, a good agreement is obtained in the off-diagonal component of the dielectric tensor for the continuous polycrystalline Co sample [Fig. 6(b)]. On the other hand some differences are found in the diagonal component [Fig. 6(a)], especially in the imaginary part due to interband contributions not being considered.

In granular layers, such as the ones studied here, we should expect that some of these Drude parameters might change, in particular, the relaxation time of the electrons, related to the electron mean free path ${ }^{28}$ which is considerably reduced due to the breaking of the lattice periodicity and the collisions of the electrons with the surface of the nanoparticles. The dependence of the relaxation time on the size of the nanoparticles can be expressed as ${ }^{2,28,29}$
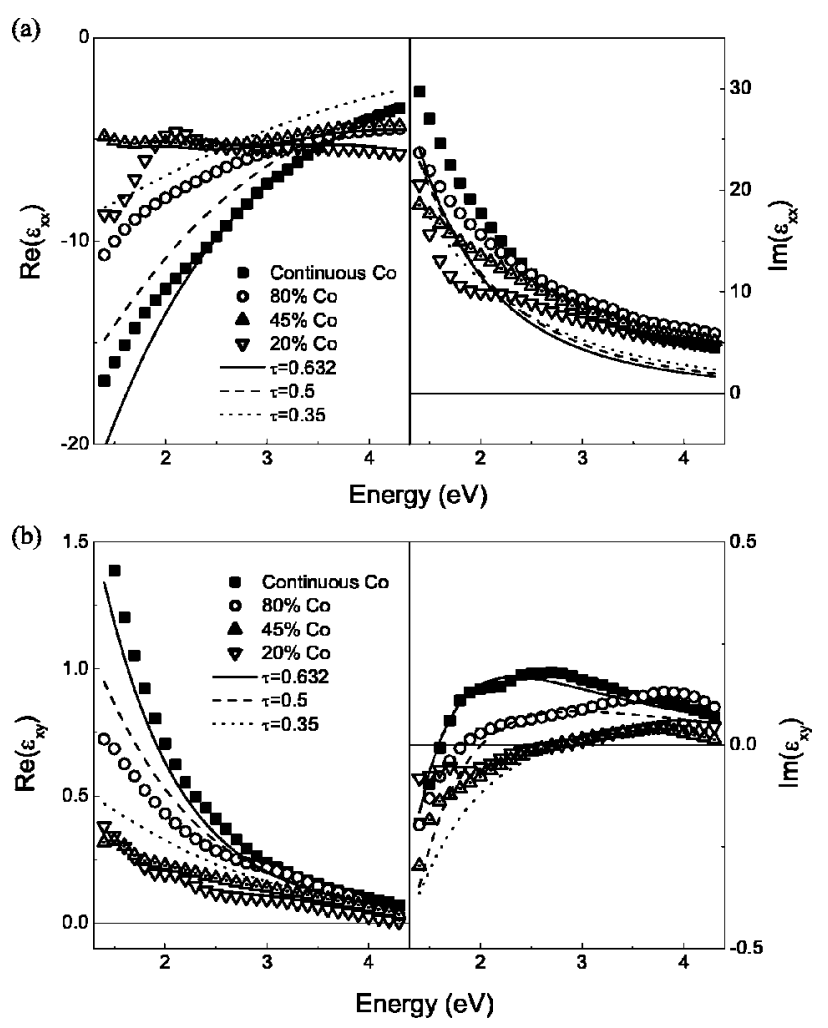

FIG. 6. Real and imaginary parts of the diagonal (a) and off-diagonal (b) components of the dielectric tensor of Co being part of the $20 \%, 45 \%$, and $80 \%$ samples, together with those measured in a polycrystalline Co film.

$$
\frac{1}{\tau}=\frac{1}{\tau_{0}}+\frac{A}{R}
$$

where $R$ is the radius of the nanoparticle and $A$ is a constant that depends on the material and on the shape of the nanoparticles. A decrease of the relaxation time as the size of the nanoparticles decreases is therefore expected. In the case of the $80 \%$ sample, it was observed by TEM that the nanoparticles are forming large aggregates of nanoparticles, with a high concentration of $\mathrm{Co}$ in the layer. In fact, the dielectric tensor is similar to the polycrystalline sample and it is only necessary to decrease the relaxation time from $\tau$ $=0.632$ to $0.5 \mathrm{eV}^{-1}$ keeping the rest of the Krinchik parameters for Co constant in order to reproduce the evolution, as can be observed in Figs. 6(a) and 6(b) (dashed lines). Again some differences are found in the real and, in particular, in the imaginary parts of the diagonal dielectric tensor due to interband transitions not being considered in this model. On the other hand, the dielectric tensor of the $45 \%$ and $20 \%$ samples is very similar, even taking into account that the $45 \%$ sample is close to the percolation threshold. This indicates that the size of the nanoparticles is very similar in these samples. To properly fit the dielectric tensor for these granular films it is necessary to decrease the relaxation time of Co up to $\tau=0.35 \mathrm{eV}^{-1}$ [dotted line in Figs. 6(a) and 6(b)], obtaining again a good agreement in the off-diagonal component, whereas some differences due to interband contributions are found in the diagonal components. As can be observed we can reproduce the evolution of dielectric tensor of Co in the nanoparticles assuming that the size of the nano- 
particles determines the relaxation time of the electrons.

This result suggests that, as far as the off-diagonal component, the dielectric tensor for polycrystalline Co and nanoparticle layers can be described very accurately by using a Drude model with no interband contributions and by only varying the relaxation time of the electrons as a function of the nanoparticle size. Moreover, in the case of crystalline Co fcc and hcp phases reported by Weller et al., ${ }^{30}$ small differences are found between the off-diagonal components of both phases and those of polycrystalline Co, which may suggest that, as far as this component is concerned, the main contribution is related with the intraband transition. On the other hand, there are differences in the diagonal components of polycrystalline Co and the Drude fitting in Fig. 6(a) (continuous lines), especially in the imaginary part. These differences can be ascribed to interband contributions not considered here that have been found to be more important in the imaginary part. $^{31}$ In this case significant differences are found between polycrystalline and crystalline Co samples. In the latter a strong transition centered at $0.7 \mathrm{eV}$ (Ref. 32) is detected, which does not appear in the polycrystalline case. These facts point out that, although the intraband contribution is the most important also in the diagonal component, the interband contributions are more evident in this case, mainly in systems with high crystalline quality.

\section{CONCLUSIONS}

The optical and MO properties of Co nanoparticles embedded in a $\mathrm{ZrO}_{2}$ matrix have been studied. The samples were grown by laser ablation with different Co concentrations. Narrow particle size distributions were obtained, remaining almost constant $(D=2.5 \mathrm{~nm})$ for concentrations below the percolation threshold and broadening as the percolation is approached. Above percolation, large aggregates are found. The optical and MO response of the granular layers fundamentally depend on the concentration and size of the Co nanoparticles. The evolution of the optical and MO response of the layers as a function of the concentration of Co has been described using an effective medium approximation. We have observed changes in the dielectric tensor of continuous $\mathrm{Co}$ and nanoparticle systems above and below the percolation threshold, due to the different sizes of the aggregates and nanoparticles within them. These changes are explained by means of a free electron Drude model and are related to a decrease of the relaxation time of the electrons in the Co nanoparticles induced by a reduction of the electron mean free path as the size of the nanoparticles is reduced.

\section{ACKNOWLEDGMENTS}

This work was partially financed by the Spanish Commission of Science and Technology (Comunidad de Madrid),
Spanish CICYT (MAT2003-01124), and Catalan DURSI (2005SGR00969). Two of the authors (C.C.) and (Z.K.) acknowledge the Ministerio de Educación y Ciencia through FPI and the Juan de la Cierva program for financial support. The authors thank Professor M. Varela for his support in the sample preparation and R. Serna for help with the ellipsometry measurements.

${ }^{1}$ B. Kalska, J. J. Paggel, P. Fumagalli, H. Hilgendorff, and M. Giersig, J. Appl. Phys. 92, 7481 (2002).

${ }^{2}$ N. Del Fatti, F. Vallée, C. Flytzanis, Y. Hamanaka, and A. Nakamura, Chem. Phys. 251, 215 (2000).

${ }^{3}$ F. Luis et al., Phys. Rev. B 65, 094409 (2002).

${ }^{4}$ F. Delogu, Nanotechnology 17, 2027 (2006).

${ }^{5}$ R. Carey, D. M. Newman, and B. W. J. Thomas, Thin Solid Films 129, 231 (1985)

${ }^{6}$ M. F. Luo, C. I. Chiang, H. W. Shiu, S. D. Sartale, and C. C. Kuo, Nanotechnology 17, 360 (2006).

${ }^{7}$ J. Inoue and S. Maekawa, Phys. Rev. B 53, R11927 (1996).

${ }^{8}$ B. J. Hattink, M. García del Muro, Z. Konstantinović, X. Batlle, A. Labarta, and M. Varela, Phys. Rev. B 73, 045418 (2006).

${ }^{9}$ L. T. Nguyen, F. D. Tichelaar, and J. C. Lodder, J. Magn. Magn. Mater. 290, 1294 (2005).

${ }^{10}$ A. Lisfi and J. C. Lodder, Phys. Rev. B 63, 174441 (2001).

${ }^{11}$ S. Ohnuma, H. Fujimori, S. Mitani, and T. Masumoto, J. Appl. Phys. 79, 5130 (1996).

${ }^{12}$ W. S. Kim, M. Aderholz, and W. Kleemann, Meas. Sci. Technol. 4, 1275 (1993).

${ }^{13}$ C. Clavero, A. Cebollada, G. Armelles, Y. Huttel, J. Arbiol, F. Peiró, and A. Cornet, Phys. Rev. B 72, 024441 (2005).

${ }^{14}$ S. Sankar, A. E. Berkowitz, and D. J. Smith, Phys. Rev. B 62, 14273 (2000).

${ }^{15}$ R. H. Kodama and A. S. Edelsetein, J. Appl. Phys. 85, 4316 (1999).

${ }^{16}$ O. Kitakami, H. Sato, Y. Shimada, F. Sato, and M. Tanaka, Phys. Rev. B 56, 13849 (1997).

${ }^{17}$ V. Dureuil, C. Ricolleau, M. Gandais, and C. Grigis, Eur. Phys. J. D 14, 83 (2001).

${ }^{18}$ C. Clavero, A. Cebollada, G. Armelles, Y. Huttel, J. Arbiol, F. Peiró, A. Cornet, and C. Domínguez, Proc. SPIE 5838, 266 (2005).

${ }^{19}$ M. Schubert, Phys. Rev. B 53, 4265 (1996).

${ }^{20}$ B. Sepulveda, Y. Hutter, C. M. Boubeta, A. Cebollada, and G. Armelles, Phys. Rev. B 68, 064401 (2003).

${ }^{21}$ D. Stroud, Phys. Rev. B 12, 3368 (1975).

${ }^{22}$ J. L. Menéndez, B. Bescós, G. Armelles, R. Serna, J. Gonzalo, R. Doole, A. K. Petford-Long, and M. I. Alonso, Phys. Rev. B 65, 205413 (2002).

${ }^{23}$ J. S. Ahn, K. H. Kim, T. W. Noh, D.-H. Riu, K. H. Boo, and H.-E. Kim, Phys. Rev. B 52, 15244 (1995).

${ }^{24}$ P. B. Johnson and R. W. Christy, Phys. Rev. B 6, 4370 (1972).

${ }^{25}$ T. K. Xia, P. M. Hui, and D. Stroud, J. Appl. Phys. 67, 2736 (1990).

${ }^{26}$ G. S. Krinchik, J. Appl. Phys. 35, 1089 (1964).

${ }^{27}$ R. Carey and B. W. J. Thomas, J. Phys. D 7, 2362 (1974).

${ }^{28}$ U. Kreibing and M. Vollmer, Optical Properties of Metal Clusters (Springer, Berlin, 1995).

${ }^{29}$ S. Norrman, T. Andersson, C. G. Granqvist, and O. Hunderi, Phys. Rev. B 18, 674 (1978).

${ }^{30}$ D. Weller, G. R. Harp, R. G. C. Farrow, A. Cebollada, and J. Sticht, Phys. Rev. Lett. 72, 2097 (1994).

${ }^{31}$ A. Delin, S. Auluck, O. Eriksson, B. Johansson, and J. M. Wills, Phys. Rev. B 60, 14105 (1999).

${ }^{32}$ G. Y. Guo and H. Ebert, Phys. Rev. B 50, 10377 (1994). 